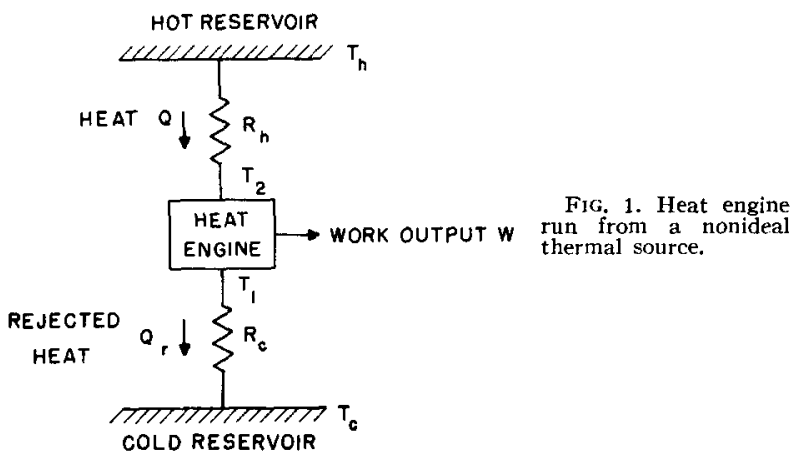

Fig. 1, in which the heat engine shown is assumed to be a thermoelectric generator.

An electrical analogy of the nonideal heat reservoir is a voltage source $e$ with a finite series resistance $r$. The fact that this source has a maximum amount of power that can be drawn from it (the "available power" $\left.e^{2} / 4 r\right)$, suggests that the nonideal thermal source may have a similar maximum. We have calculated this "available power," which cannot be achieved with practical thermoelectric generators, because of associated irreversible effects (joule heating and heat conduction).

Observe from Fig. 1 that the heat engine is limited by the Carnot efficiency, so the useful work output $W$ is given by

$$
W \leq\left[\left(T_{2}-T_{1}\right) / T_{2}\right] Q,
$$

where the equality sign holds only for reversible (Carnot) engines. When $Q$ is small, the work output is small. On the other hand, suppose $Q$ is large. Then there is an appreciable temperature drop across the thermal resistances and $\left(T_{2}-T_{1}\right)$ is small, so that, again, the useful work output is small. A maximum value of $W$ is obtained for reversible heat engines, and for an intermediate value of $Q$.

To find this maximum value for $W$, we use the equations that relate $T_{2}$ and $T_{1}$, which are the temperatures of the heat engine, to $T_{h}$ and $T_{c}$, the reservoir temperatures:

$$
\begin{aligned}
T_{1} & =T_{c}+Q_{c} R_{c} \\
& =T_{c}+Q R_{c}-W R_{c} \\
T_{2} & =T_{h}-Q R_{h} .
\end{aligned}
$$

Substituting Eqs. (2) and (3) in the efficiency expression of Eq. (1), we solve for $W$. We find that $R_{h}$ and $R_{c}$ appear only together as a sum; if this total thermal resistance is

then

$$
R=R_{h}+R_{c}
$$

$$
W \leq\left[\left(T_{h}-T_{c}-Q R\right) /\left(T_{h}-Q R\right)\right] Q .
$$

Observe that in Eq. (5), the equality sign holds if the heat engine is reversible; otherwise the inequality sign holds. The right-hand side has a maximum that occurs when $Q$ is

$$
Q_{\mathrm{opt}}=\left[T_{h}-\left(T_{h} T_{\mathrm{c}}\right)^{i}\right] / R \text {. }
$$

The useful work output, the heat input, and the heat rejected under optimum conditions, with a Carnot engine, are:

$$
\begin{aligned}
& W_{\text {opt }}=\frac{T_{h}-T_{c}}{R} \frac{\left(T_{h}\right)^{t}-\left(T_{c}\right)^{t}}{\left(T_{h}\right)^{t}+\left(T_{c}\right)^{t}} \\
& Q_{\mathrm{opt}}=\frac{T_{h}-T_{c}}{R} \frac{\left(T_{h}\right)^{1}}{\left(T_{h}\right)^{3}+\left(T_{c}\right)^{3}}
\end{aligned}
$$

and

$$
\left(Q_{\tau}\right)_{\mathrm{opt}}=Q_{\mathrm{opt}}-W_{\mathrm{opt}}=\frac{T_{h}-T_{c}}{R} \frac{\left(T_{c}\right)^{\xi}}{\left(T_{h}\right)^{\frac{1}{t}}+\left(T_{c}\right)^{*}} .
$$

Furthermore, under these optimized conditions, the efficiency is

$$
\eta_{\mathrm{opt}}=\frac{W_{\mathrm{opt}}}{Q_{\mathrm{opt}}}=\frac{\left(T_{h}\right)^{\mathrm{t}}-\left(T_{\mathrm{c}}\right)^{t}}{\left(T_{h}\right)^{t}} .
$$

Equations (1) and (10) show that under optimized conditions,

$$
\left(T_{2} / T_{1}\right)_{\mathrm{opt}}^{2}=T_{h} / T_{c} \text {. }
$$

Note that the first factor in the expressions for $W_{\text {opt }}, Q_{\text {opt }}$, and $\left(Q_{r}\right)_{\text {opt }}$ is the heat that would flow between the reservoirs if the heat engine were replaced by an infinitely conducting path; that is, it is the "short-circuit heat flow."

These expressions are interesting in that only the total resistance between the reservoirs appears; thus, as far as these fundamental limits are concerned, it does not matter whether all of the resistance is at one reservoir, or is split between them. Also, note that the efficiency under optimum power-output conditions is independent of $R$. Since all physical sources may be expected to have some finite thermal resistance, it appears that if a Carnot engine is operated between practical heat reservoirs in such a way as to have a maximum power output, then the efficiency must be reduced, in accordance with $\mathrm{Eq}$. (10), from the Carnot efficiency calculated from the reservoir temperatures, regardless of the size of the thermal resistances.

I wish to thank Professor P. E. Gray, who made valuable comments on this work.

* This work was supported in part by a Ford Foundation Fellowship; and in part by the U.S. Army Signal Corps, the Air Force Office of Scientific Research, and the Office of Naval Research.

1 P. E. Gray, Trans. Am. Inst. Elec. Engrs. Part I, 79, No. 47, 15 (1960)

\section{Equation of Motion of the Torsion Pendulum and the Complex Modulus}

\author{
N. W. TSChoEgL \\ Bread Research Institute of Australia, North Ryde, \\ New South Wales, Australia \\ (Received March 9, 1961)
}

TN a paper published several years ago, Nielsen ${ }^{1}$ made an erroneous attempt to express the differential equation describing the motion of the simple torsion pendulum, operating in free oscillation, in terms of the complex shear modulus. This treatment has since been adopted in a number of other publications ${ }^{2-4}$ the last mentioned having appeared quite recently in this journal. Nielsen wrote

$$
I\left(d^{2} \theta / d t^{2}\right)+k G^{*} \theta=0,
$$

where $I$ is the moment of inertia, $G^{*}$ the complex modulus, $k$ a shape constant, and $\theta$ is the angle of twist.

Substituting the equation for the angle of twist of the freely oscillating torsion pendulum,

$$
\theta=\theta_{0} \exp (j \omega-\alpha) t
$$

where $\omega$ is the radian frequency of the oscillation and $\alpha$ is the attenuation factor into Eq. (1), he arrived at the expression

$$
G^{\prime}=I\left(\omega^{2}-\alpha^{2}\right) / k
$$

for the real part of the complex modulus $G^{*}$. However, the correct relation is known to be

$$
G^{\prime}=I\left(\omega^{2}+\alpha^{2}\right) / k
$$

This can be derived readily by substituting Eq. (2) into the usual form of the differential equation for the freely oscillating torsion pendulum

$$
I\left(d^{2} \theta / d t^{2}\right)+k \eta^{\prime}(d \theta / d t)+k G^{\prime} \theta=0,
$$

where $\eta^{\prime}$ is the real part of the complex viscosity.

It is then seen that Eq. (1) incorrectly assumes that one may substitute $j \omega k \eta^{\prime}$, or $j k G^{\prime \prime}$, for the middle term of Eq. (5) while the correct substitution is $(j \omega-\alpha) k \eta^{\prime}$, or $(j-\alpha / \omega) k G^{\prime \prime}$. If, therefore, Eq. (5) is to be written in terms of the complex modulus, it must take the form

or

$$
\begin{gathered}
I\left(d^{2} \theta / d t^{2}\right)+k\left(G^{*}-\alpha \eta^{\prime}\right) \theta=0 \\
I\left(d^{2} \theta / d t^{2}\right)+k\left(G^{*}-\alpha G^{\prime \prime} / \omega\right) \theta=0 .
\end{gathered}
$$


It should be noted that, in case of forced oscillation, the equation

$$
I\left(d^{2} \theta / d t^{2}\right)+k G^{*} \theta=\Theta \exp j \omega^{\prime} t,
$$

where $\omega^{\prime}$ is the impressed radian frequency and $\Theta$ is the torque, will lead to the correct particular integral as may be seen by substituting the steady-state solution

$$
\theta=\theta^{\prime} \exp j\left(\omega^{\prime} t-\phi\right),
$$

into $\mathrm{Eq}$. (7). This is so since the substitution of $j \omega^{\prime} k \eta^{\prime}$, or $j k G^{\prime \prime}$, for the term containing the first derivative, is valid for the steadystate solution. However, the complete integral containing the transient solution cannot be obtained from Eq. (7).

\footnotetext{
1 L. E. Nielsen, Rev. Sci. Instr. 22, 690 (1951).
2 R. Buchdahl, in Rheology, edited by F. Eirich (Academic Press, Inc., $2 \mathrm{R}$. Buchdahl, in Rheology, edited by
New York, 1958), Vol. 2, pp. 148-150.

L. E. Nielsen, R. Wall, and G. Adams, J. Colloid Sci. 13, 441 (1958).

4 D. G. Flom, J. Appi. Phys. 31, 310 (1960).
}

\section{Friction of Molybdenum Diselenide*}

Melvin T. Lavik, T. Bruce Daniel, and A. Neal Abbott Midwest Research Instilute, Kansas Cily 10, Missouri

(Received May 4, 1961)

$\mathbf{T}$ HE friction of lamellar solids is markedly influenced by adsorbed vapors. For example, the slickness normally associated with graphite depends on moisture adsorbed from the air. $\mathrm{MoS}_{2}$ and $\mathrm{WS}_{2}$ friction, on the other hand, is least in vacuum and increases in the presence of air, water vapor, nitrogen, argon, and helium. Furthermore, in vacuum $\mathrm{MoS}_{2}$ and $\mathrm{WS}_{2}$ exhibit transient frictional characteristics that are not observed in the presence of an atmosphere or with graphite either in vacuum or with an atmosphere present.1,2 As pellets of compressed $\mathrm{MoS}_{2}$ or $\mathrm{WS}_{2}$ powder are rubbed against a circular metal plate, a continuous track of pellet material builds up on the plate until the coefficient of friction $\mu$ reaches an equilibrium value $\mu_{e}$ corresponding to pellet material sliding against itself. If a pellet which has been sliding in vacuum is allowed to stand in vacuum, then, upon resumption of sliding, the initial coefficient of friction $\mu_{i}$ will be higher than $\mu_{e}$. Continued sliding reduces $\mu$ to $\mu_{e}$. $\left(\mu_{i}-\mu_{e}\right)$ increases with stop time $T$ but reaches a plateau for $T$ greater than about 4 hr. This behavior has been explained by the assumption that sulfur is liberated during sliding and forms an adsorbed layer on the surfaces. ${ }^{1,3}$ The adsorbed sulfur layer is presumed to play the same role in $\mathrm{MoS}_{2}$ and $\mathrm{WS}_{2}$ friction that water vapor plays in graphite friction. Evaporation of the sulfur during the stop time would account for the difference between $\mu_{i}$ and $\mu_{e}$.

Compounds similar to $\mathrm{MoS}_{2}$ can be employed to test the adsorbed sulfur theory. $\mathrm{MoSe}_{2}$ has the same crystal form as $\mathrm{MoS}_{2}$ and in air at room temperature both have essentially the same friction coefficient. However, the vapor pressure of bulk selenium is much less than the vapor pressure of bulk sulfur. Our initial supposition was that an adsorbed selenium film would evaporate more slowly from $\mathrm{MoSe}_{2}$ than would an adsorbed sulfur film evaporate from $\mathrm{MoS}_{2}$. Extension of the adsorbed sulfur theory to $\mathrm{MoSe}_{2}$ suggested that $\left(\mu_{i}-\mu_{e}\right)$ should increase more slowly with $T$ than it does for $\mathrm{MoS}_{2}$ friction.

We have measured the friction of $\mathrm{MoSe}_{2}$ in vacuum $\left(10^{-9} \mathrm{~atm}\right)$ using apparatus which has been described in detail elsewhere." Since pump oil vapor is known to increase $\mathrm{MoS}_{2}$ friction, we only need point out here that a large dry ice-acetone cold trap was placed between pump and chamber. Data were taken for 11 stop time values ranging from $5 \mathrm{~min}$ to $695 \mathrm{hr}$. In all of the experiments, the pellet load was $132 \mathrm{~g}$ and the sliding speed $310 \mathrm{~cm} / \mathrm{sec}$. The nominal pressure at the sliding interface, computed from the apparent area of contact, was $10^{4} \mathrm{~d} / \mathrm{cm}^{2}$. Figure 1 shows the essential features of $\mathrm{MoSe}_{2}$ friction. To avoid crowding, only 4 curves are included.

In vacuum the" mean value of $\mu_{e}$ is 0.108 with a standard deviation of 0.004 . (Cf. $\mu_{e}=0.065$ for $\mathrm{MoS}_{2}$ under identical conditions.) In air or helium $\mu_{e}$ is about twice the vacuum value

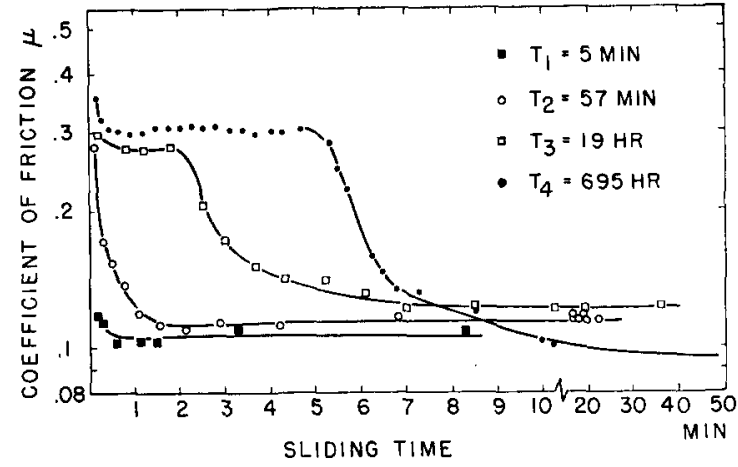

FIG. 1. Coefficient of friction of MoSen sliding in vacuum against MoSez after standing in vacuum for stop times $T$. The curve for $T=695$ hr reaches $\mu=0.09$ after $80 \mathrm{~min}$ sliding (point not shown).

as is the case for $\mathrm{MoS}_{2}$ friction. Contrary to our initial expectation, the dependence of $\left(\mu_{i}-\mu_{e}\right)$ on $T$ is very nearly the same as for $\mathrm{MoS}_{2}$, at least for $T<2 \mathrm{hr}$. For $T>2 \mathrm{hr}$ there is a qualitative change in frictional behavior. $\mu$ does not decrease smoothly from $\mu_{i}$ to $\mu_{e}$ but pauses at an intermediate value $\mu_{m} \approx 0.3$. It remains at this value for a short time, then decreases to $\mu_{e}$. The duration of the pause at $\mu_{m}$ increases almost linearly with stop time. This behavior suggests the presence of a contaminant film which is removed by sliding. Such an effect could result from leakage of pump oil past the cold trap although in the same apparatus $\mathrm{MoS}_{2}$ does not show similar behavior even after a 192-hr stop time.

In addition to the experiments just described, we investigated the friction of single crystals of $\mathrm{MoS}_{2}$ (natural molybdenite). The question had arisen whether the behavior reported for $\mathrm{MoS}_{2}$ and $\mathrm{WS}_{2}$ could be accounted for, at least to some extent, by a property of compressed powders. The single-crystal experiments, however, gave the same results as experiments previously done with compressed $\mathrm{MoS}_{2}$ powder at room temperature in vacuum and in atmospheres of air, nitrogen, and helium. The one discrepancy was that the single crystals would not deposit a track in vacuum, their shear strength being so small that they wore away almost immediately. On the other hand, if the initial sliding was done in air, a satisfactory track built up and subsequent sliding in vacuum caused very little wear. All of the data were taken with the crystals oriented so that the basal planes made an angle of about $10^{\circ}$ with the plane of sliding.

Further experiments that would help interpret the frictional behavior of $\mathrm{MoS}_{2}$-type solids include measurement of adsorption energies for sulfur on $\mathrm{MoS}_{2}$ and selenium on $\mathrm{MoSe}_{2}$ and an investigation of $\mathrm{MoTe}_{2}$ friction. More to the point, however, would be observation of the rubbing surfaces for direct evidence of adsorbed films.

* This work was supported by Materials Central, Wright Air Development Division, United States Air Force.

1 V. R. Johnson and G. W. Vaughn, J Appl. Phys. 27, 1173 (1956).

2 V. R. Johnson, M. T. Lavik, and G, W. Vaughn. J. Appl. Phys. 28, 821 (1957). M. T. 246 (1959).

V. R. Johnson, G. W. Vaughn, and M. T. Lavik, Rev. Sci. Instr. 27, 611 (1959).

\section{Similarity Function for Pattern Recognition DAN MCLACHLAN, JR. Stanford Research Institute, Menlo Park, California (Received April 10, 1961)}

GINCE many of the aspects of mechanical, optical, and electrical $\mathcal{O}$ machines for information retrieval, translation of languages, and data processing are dependent upon sensing devices which are capable of recognizing patterns, much could be gained from having on hand a mathematical function which expresses the identity of, or the similarity between, two patterns on a plane. 